D

Dipartimento di Elettronica, Informazione e Bioingegneria, Politecnico di

Milano, Milan, Italy

\title{
Respiratory physiology of pregnancy
}

\section{Physiology masterclass}

During healthy pregnancy, pulmonary function, ventilatory pattern and gas exchange are affected through both biochemical and mechanical pathways, as summarised in figure 1 .

\section{Chemical/hormonal changes}

During pregnancy, the physiological alteration of hormonal patterns is the main cause of ventilatory changes in respiratory function.

\section{Progesterone}

Progesterone gradually increases during the course of pregnancy, from $25 \mathrm{ng} \cdot \mathrm{mL}^{-1}$ at 6 weeks' to $150 \mathrm{ng} \cdot \mathrm{mL}^{-1}$ at 37 weeks' gestation [1-5]. Progesterone acts as trigger of the primary respiratory centre by increasing the sensitivity of the respiratory centre to carbon dioxide, as indicated by the steeper slope of the ventilation curve in response to alveolar carbon dioxide changes [6]. Progesterone alters the smooth muscle tone of the airways resulting in a bronchodilator effect. It also mediates hyperaemia and oedema of mucosal surfaces, causing nasal congestion.

\section{Oestrogen}

The circulating levels of oestrogen increase during pregnancy, before or in parallel to those of progesterone. Oestrogen is a mediator of progesterone receptors. It increases the number and the sensitivity of progesterone receptors within the hypothalamus and medulla, the central neuronal respiratory-related areas [1].

\section{Prostaglandins}

Prostaglandins stimulate uterine smooth muscle during labour and are present during all three trimesters of pregnancy. Prostaglandin $\mathrm{F}_{2 \alpha}$ increases airway resistance by bronchial smooth muscle constriction, whereas a bronchodilator effect can be a consequence of prostaglandins $E_{1}$ and $E_{2}[1]$.

\section{Mechanical effects}

The progressive uterine distension is the major cause of lung volume and chest wall changes during pregnancy, which comprise elevation of the diaphragm and altered thoracic configuration [1-3]. The enlarging uterus increases the end-expiratory abdominal (gastric) pressure $(P$ ga), thereby displacing the diaphragm upwards, with two consequences [2]. First, the negative pleural pressure (oesophageal pressure $\left(P_{\text {oes }}\right)$ ) increases, leading to an earlier closure of the small airways with consequent reduction of functional residual capacity (FRC) and expiratory reserve volume (ERV) [1, 3, 4, 7]. Secondly, the chest height becomes shorter, but the other thoracic dimensions increase in order to maintain constant total lung capacity (TLC) [1, 2, 7].
Cite as: LoMauro A, Aliverti A. Respiratory physiology of pregnancy. Breathe 2015: 11: 297-301. 
$\uparrow$ Progesterone

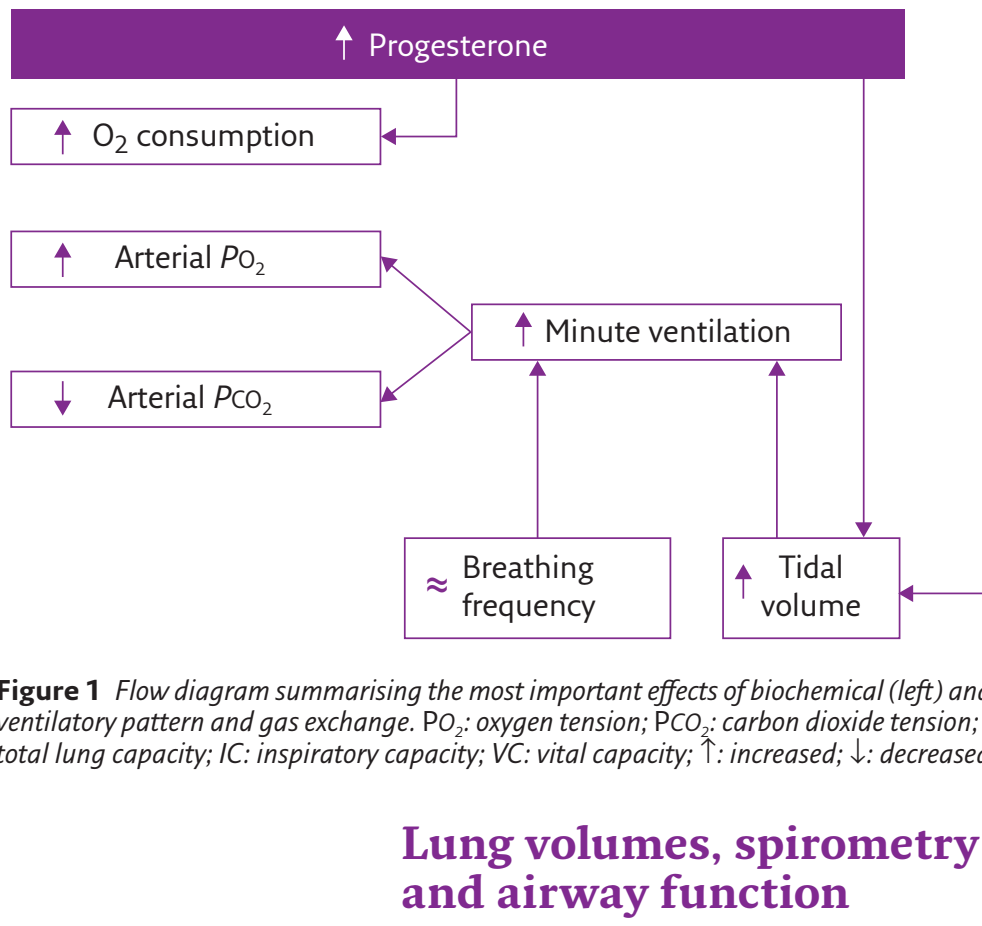

Throughout pregnancy, spirometry remains within normal limits, with forced vital capacity (FVC) [1, 3-5, 7-9], forced expiratory volume in $1 \mathrm{~s}$ (FEV1) $[1,4,5,7,9]$ and peak expiratory flow [4, 7-9] not changing or modestly increasing with unaltered FEV1/FVC index [1, 4, 5, 7, 9].

Conversely, lung volumes undergo major changes: ERV gradually decreases during the second half of pregnancy (reduction of $8-40 \%$ at term) because residual volume reduces (by 7-22\%). FRC then decreases (by 9.5-25\%) while inspiratory capacity increases at the same rate in order to maintain stable TLC [1, 3, 4, 7].

Respiratory resistance increases while respiratory conductance decreases during pregnancy. Total pulmonary and airways resistances tend to decrease in late pregnancy as a consequence of hormonally induced relaxation of tracheobronchial tree smooth muscles [1, 4, 8]. Pulmonary static and dynamic compliance, diffusing capacity and static lung recoil pressure do not change during pregnancy $[1,2,4,7]$. Respiratory function does not differ between singleton and twin pregnancies [7].

\section{Chest wall geometry and displacement}

The pregnancy-induced reduction of FRC is accompanied not only by abdominal enlargement but also by ribcage dimension increases. The ribcage expands, as its transversal diameter and the lower thoracic perimeter increase in the third trimester compared with postpartum. The average subcostal angle of the ribs at the xiphoidal level increases from $68.5^{\circ}$ at the beginning of pregnancy to $103.5^{\circ}$ at term [1-3].
Such ribcage dimension changes could be a consequence of fatty tissue and fluid accumulation, particularly blood, since pulmonary blood volume is often increased in pregnancy. However, it seems more likely to be a compensation of the shortening of the thorax due to the upward movement of the diaphragm, in order to guarantee space to the lung and preserve TLC.

Chest wall compliance decreases in late pregnancy due to the increased abdominal content [10].

\section{Breathing pattern at rest}

Minute ventilation $\left(V^{\prime} E\right)$ starts to increase significantly (by up to $48 \%$ ) during the first trimester of gestation, due to higher tidal volume $(V T)$ with unchanged respiratory rate. This ventilatory pattern is then maintained throughout the course of pregnancy $[1-3,5,8]$.

Both $V$ T/inspiratory time ratio and mouth occlusion pressure at $100 \mathrm{~ms}$ increase during pregnancy, indicating respectively that the ventilatory and inspiratory drives augment $[2,8]$.

The increased $V T$ in pregnancy is achieved mainly by an improved displacement of the ribcage with no consistent changes in the abdominal contribution measured with magnetometers [2, 3].

\section{Respiratory muscles}

Maximal inspiratory and expiratory pressures do not change during pregnancy or after delivery indicating that, although there is alteration of chest wall geometry, the respiratory muscle strength is preserved [2-4, 11].

With pregnancy progression, the resting position of the diaphragm moves $5 \mathrm{~cm}$ upward with the increasing uterus size, as shown by chest radiograph measurement $[1,2,12]$. This causes 
the following changes to the diaphragm: its capability to generate tension increases secondary to muscle fibre lengthening; its area of apposition to the lower ribcage increases; and its radius of curvature increases, due to the progressive enlargement of the lower ribcage to give space to the lungs. In addition, the upward movement of the diaphragm causes FRC decrease.

The inspiratory movements of the diaphragm are similar or become even broader than postpartum [1, 12], and trans-diaphragmatic pressure swings during tidal breathing do not change [2]. The diaphragmatic work may increase as a consequence to contract against higher load represented by higher end-expiratory $P_{\text {ga }}$ and enlarged gravid uterus. This hypothesis is supported by the tension time index of the diaphragm, which falls after delivery [2].

During pregnancy, chest wall expansion is shifted toward the ribcage because of an enhanced coupling between abdominal pressure and the lower ribcage $[2,3]$. Thanks to the increased area of apposition, in fact, the abdominal pressure generated by the contraction of the diaphragm acts mainly on the lower ribs, thereby elevating and expanding the ribcage where the diaphragm is apposed.

Two possible respiratory muscle strategies can be considered during pregnancy. 1) Higher inspiratory intercostal and accessory muscle recruitment, since the increased thoracic volume displacement and pleural pressure swings could also be a consequence of their enhanced action $[2,3]$. 2) Similar relative contribution between the diaphragm and the inspiratory intercostal muscles, since the slope of the Pga versus Poes curve remains constant $[2,3]$.

The progressive increment of the anterior abdominal dimension leads to morphological adaptation of the abdominal muscles by lengthening their fibres up to $115 \%$, changing their line of action, altering their angle of insertion and reducing their thickness. The consequences are compromised functional ability, poor torque production and reduced ability to stabilise the pelvis against resistance. The latter may be implicated in back pain during pregnancy [13, 14].

\section{Oxygen consumption, arterial blood gases and acid-base status}

Oxygen consumption and basal metabolic rate increase (by up to $21 \%$ and $14 \%$, respectively), but to a lesser extent than ventilatory augmentation [1, 5]. Pregnancy-induced reduction in FRC combined with a rise in oxygen consumption lowers the oxygen reserve of the mother.

As a consequence of $V^{\prime} E$ increment, alveolar and arterial carbon dioxide tension $\left(\mathrm{PCO}_{2}\right)$ levels decrease to plateaux around 27 and $32 \mathrm{mmHg}$, respectively [1, 4, 5]. During labour, arterial $P \mathrm{CO}_{2}$ further decreases with each contraction, while at full dilatation it decreases even between contractions [1].

Following hyperventilation and reduced levels of $\mathrm{PCO}_{2}$, arterial oxygen tension increases, reaching $106-108 \mathrm{mmHg}$ and $101-104 \mathrm{mmHg}$ in the first and third trimesters, respectively [1].

Despite hyperventilation and respiratory alkalosis, blood $\mathrm{pH}$ remains almost constant at slightly alkalotic values (7.40-7.47), thanks to renal compensation by increasing the excretion of bicarbonate $[1,4,5]$.

\section{Dyspnoea}

Dyspnoea or breathlessness is commonly reported by $70 \%$ of healthy pregnant women during their daily living activities, starting from the very first trimester of gestation. Such an early time of onset excludes the pregnancy-induced mechanical changes from playing a role in the genesis of the symptoms [1]. Possible explanations could be: 1) an increased awareness of the new sensation of the physiological hyperventilation associated with pregnancy; 2) an increased central perception of respiratory discomfort with increasing $V^{\prime} \mathrm{E}$; or 3 ) a combination of these two.

Recently, pregnancy-induced hyperventilation has been demonstrated to be the result of complex interactions between changes in chemoreflex drives, acid-base balance, metabolic rate and cerebral blood flow [5]. It is therefore a difficult challenge for the caregiver to distinguish between physiological and pathophysiological origins of dyspnoea.

\section{Exercise}

Pregnant women preserve their aerobic working capacity even in late gestation. The physiological response to incremental exercise in healthy pregnant women consists of augmentation of both $V^{\prime} E$ and oxygen consumption, with higher ventilatory equivalent (i.e. V'E/oxygen consumption) [1, 4].

Hyperventilation occurs thanks to the recruitment of resting inspiratory capacity combined with pregnancy-induced bronchodilation, allowing $V T$ to increase in order to meet the metabolic demands. Inspiratory capacity is recruited thanks to stable TLC and pre-exercise end-expiratory lung volume reduction. Because ERV is reduced, end-expiratory lung volume does not further decrease during exercise to avoid the mechanical disadvantage of the lower portion of the pressurevolume curve of the respiratory system.

The ratio of the contractile effort of the respiratory muscles (tidal $P_{\text {oes }}$ /maximal inspiratory $P$ oes) to the thoracic volume displacement (VT/vital capacity), 
Table 1 Principal features to consider when treating unhealthy pregnant women

\begin{tabular}{l} 
Disease \\
\hline Asthma [15] \\
Pulmonary oedem \\
[15] \\
\\
Severe restrictive \\
lung disease [10]
\end{tabular}

\section{Features and/or indications}

Recommended use of corticosteroids to prevent critical illness

Oral corticosteroids associated with a two-fold increase in pre-eclampsia and with a minimal incidence $(0.4 \%)+$ of oral clefts (if taken in the first trimester)

\section{lung disease [10]}

Pre-eclampsia [16]

\section{Heart and lung transplantation [17]}

\section{Hereditary neuromuscular disorders [18]}

\section{ARDS [19]}

\section{Mechanical ventilation [20]}

Cardiogenic basis secondary to haemodynamic factors occurring during pregnancy:

$\uparrow$ cardiac output

$\uparrow$ heart rate

$\downarrow$ systemic vascular resistance

$\downarrow$ colloid osmotic pressure

A consequence of tocolytic therapy and of pre-eclampsia

If no improvement within $24 \mathrm{~h}$ after diuresis, invasive haemodynamic monitoring and/or rapid antihypertensive therapy are required

Risk of hypoxic and hypercapnic respiratory failure because the ability to increase ventilation is limited Severely reduced vital capacity but pregnancy can be well tolerated

Minimal complication: preterm delivery with newborn needing high-dependency support

Lung function and oxygen saturation should be monitored

Supplemental oxygen and noninvasive ventilation may be required

The most common obstetric disorder, with multisystem ramifications

$\uparrow$ minute ventilation because of $\uparrow$ concentration of blood leptin (a ventilation-stimulating hormone)

$\downarrow$ vital capacity secondary to lower transverse section area of the upper airways, pharyngeal oedema and excessive weight gain with higher adipose deposition around the neck

$\downarrow$ exercise tolerance

Respiratory muscle function is not affected

Better to avoid conception within the first 1-2 years after transplantation

Potential pregnancy-related complications: prematurity, low weight at birth and postpartum graft loss

Maintenance of immunosuppression with close monitoring of cyclosporine blood levels during gestation

Accurately diagnose signs of pre-eclampsia, as it is a multi-organ disease

Identify the highest risk group according to the diaphragmatic and/or bulbar involvement of the disease

$\uparrow$ respiratory muscle load by higher airway resistance and impaired bulbar load, leading to overwhelmed respiratory muscle capacity

Hypoventilation

Monitor the respiratory and cough function

Maximise airway clearance

High aspiration risk in the third trimester because of $\uparrow$ abdominal pressure and $\downarrow$ gastro-oesophageal sphincter tone

Non-obstetric causes: sepsis, pneumonia, intracerebral haemorrhage, blood transfusion and trauma

Obstetric causes: amniotic fluid embolism, pre-eclampsia, septic abortion, retained products of conception and complication from tocolytic therapy

Management includes: prompt antibiotic therapy, conservative fluid strategy, use of mechanical ventilation and extracorporeal life support in case of refractory ARDS

Similar indications to those for non-pregnant patients, with some exceptions:

Oxygen therapy may also help fetal distress

Consider that $\mathrm{PCO}_{2}$ in pregnancy is about $30 \mathrm{mmHg}$ when interpreting arterial blood gases

Airways are narrow (consequence of mucosal surface hyperaemia), so it is preferable to perform endotracheal intubation via oral route using a smaller tube

$\downarrow$ oxygen reserve of the mother, so use pre-oxygenation with $100 \%$ oxygen during intubation to avoid arterial desaturation after a short period of apnoea

Better to avoid respiratory alkalosis as it leads to problems in uterine blood flow and fetal oxygenation

Treat respiratory acidosis with bicarbonate

ARDS: acute respiratory distress syndrome; $\uparrow:$ increased; $\downarrow$ : decreased. 
being an index of neuromechanical (un)coupling of the respiratory system, does not change with increasing exercise. Pregnancy therefore does not influence the symptom-limited perceptual reasons to stop exercise and the increased breathlessness is a normal consequence of the increment of both $V^{\prime} E$ and work of breathing [4].

\section{Pregnancy during disease}

In addition to the biochemical and mechanical factors, many illnesses produce significant negative effects on respiratory maternal (and fetal) outcomes. Table 1 summarises the principal features to consider when treating unhealthy pregnant women.

\section{Conflict of interest}

None declared.

\section{References}

1. Weinberger SE, Weiss ST, Cohen WR, et al. Pregnancy and the lung. Am Rev Respir Dis 1980; 121: 559-581.

2. Contreras G, Gutiérrez M, Beroíza T, et al. Ventilatory drive and respiratory muscle function in pregnancy. Am Rev Respir Dis 1991; 144: 837-841.

3. Gilroy RJ, Mangura BT, Lavietes MH. Rib cage and abdominal volume displacements during breathing in pregnancy. $A m$ Rev Respir Dis 1988; 137: 668-672.

4. Jensen D, Webb KA, Davies GA, et al. Mechanical ventilatory constraints during incremental cycle exercise in human pregnancy: implications for respiratory sensation. J Physiol 2008; 586: 4735-4750.

5. Jensen D, Duffin J, Lam YM, et al. Physiological mechanisms of hyperventilation during human pregnancy. Respir Physiol Neurobiol 2008; 161: 76-86.

6. Lyons HA, Antonio R. The sensitivity of the respiratory center in pregnancy and after administration of progesterone. Trans Assoc Am Physicians 1959; 72: 173-180.

7. McAuliffe F, Kametas N, Costello J, et al. Respiratory function in singleton and twin pregnancy. BJOG 2002; 109: 765-769.

8. Kolarzyk E, Szot WM, Lyszczarz J. Lung function and breathing regulation parameters during pregnancy. Arch Gynecol Obstet 2005; 272: 53-58.

9. Grindheim G, Toska K, Estensen ME, et al. Changes in pulmonary function during pregnancy: a longitudinal cohort study. BJOG 2012; 119: 94-101.

10. Lapinsky SE, Tram C, Mehta S, et al. Restrictive lung disease in pregnancy. Chest 2014; 145: 394-398.

11. Lemos A, de Souza Al, Figueiroa JN, et al. Respiratory muscle strength in pregnancy. Respir Med 2010; 104: 1638-1644.
12. McGinty AP. The comparative effects of pregnancy and phrenic nerve interruption on the diaphragm and their relation to pulmonary tuberculosis. Am J Obstet Gynecol 1938; 35: 237-248.

13. Gilleard WL, Brown JM. Structure and function of the abdominal muscles in primigravid subjects during pregnancy and the immediate postbirth period. Phys Ther 1996; 76 : 750-762

14. Weis CA, Triano JJ, Barrett J, et al. Ultrasound assessment of abdominal muscle thickness in postpartum vs nulliparous women. J Manipulative Physiol Ther 2015; 38: 352-357.

15. Campbell LA, Klocke RA. Implications for the pregnant patient. Am J Respir Crit Care Med 2001; 163: 1051-1054.

16. da Silva EG, de Godoy I, de Oliveira Antunes LC, et al. Respiratory parameters and exercise functional capacity in preeclampsia. Hypertens Pregnancy 2010; 29: 301-309.

17. Vos R, Ruttens D, Verleden SE, et al. Pregnancy after heart and lung transplantation. Best Pract Res Clin Obstet Gynaecol 2014; 28: 1146-1162.

18. Norwood F, Rudnik-Schöneborn S. 179th ENMC international workshop: pregnancy in women with neuromuscular disorders 5-7 November 2010, Naarden, The Netherlands. Neuromuscul Disord 2012; 22: 183-190.

19. Duarte AG. ARDS in pregnancy. Clin Obstet Gynecol 2014; 57: 862-870.

20. Lapinsky SE, Kruczynski K, Slutsky AS. Critical care in the pregnant patient. Am J Respir Crit Care Med 1995; 152: 427-455. 